\title{
Updated Ganymede Mosaic from Voyager and Galileo Observations
}

\author{
Elke Kersten (1), Anatoly E. Zubarev (2), Thomas Roatsch (1), and Klaus-Dieter Matz (1) \\ (1) Institute of Planetary Research, German Aerospace Center, Germany (elke.kersten@dlr.de), (2) Moscow State University \\ of Geodesy and Cartography (MIIGAiK), Moscow, Russia
}

\begin{abstract}
In preparation of the JUICE mission with the primary target Ganymede [1] we generated a new controlled version of the global Ganymede image mosaic using a combination of Voyager 1 and 2 and Galileo images. Baseline for this work was the new 3D control point network from Zubarev et al., 2016 [2], which uses the best available images from both missions and led to new position and pointing of the images.
\end{abstract}

\section{Introduction}

Voyager 1 and Voyager 2 encountered Jupiter in 1979. They took 490 Narrow Angle Camera (NAC) and Wide Angle Camera (WAC) images of Ganymede's surface with pixel scales from $470 \mathrm{~m} / \mathrm{pxl}$ down to 20 $\mathrm{km} / \mathrm{pxl}$. Galileo entered orbit around Jupiter in 1995 and flew by Ganymede 15 times to acquire 149 images $(<20 \mathrm{~km} / \mathrm{pxl})$. The new control point network of Ganymede led to higher geodetic accuracy in the data and thus created the incentive to generate a new basemap with a resolution $<1 \mathrm{~km} / \mathrm{pxl}$.

\section{Image data}

The Voyager and Galileo images were acquired under very differing illumination and viewing conditions and from different observation times, although they have been taken within a short period each. Together with the varying flyby altitudes it strongly influences the images' brightness, contrast, and resolution. Another fact is that images of Ganymede are limited, so there is barely an area covered twice with a proper resolution whereas the poles suffer from a lack of image data. To reach the highest possible coverage in the global mosaic, we selected 118 Voyager 1 and 2 images and 88 Galileo SSI images including highresolution images $(<500 \mathrm{~m} / \mathrm{pxl})$ from three close Ganymede encounters [3].

\section{Control}

The 3D control point network was developed by the use of reconstructed spacecraft ephemerides [4] and consist of 3377 control points from 213 Voyager and Galileo images. The reprojection error is about $0.01-$ 0.1 pixel and the coordinate accuracy of $78 \%$ of the control points is better than $5.0 \mathrm{~km}$.

\section{Mosaicking}

The selected images were reprocessed with the new pointing and orientation data and then reprojected into the final cylindrical equidistant projection, where the small crater Anat defines the longitude system at $232^{\circ}$ East. After artefact correction, images with similar observation times and resolutions were set together to regional mosaics. The most work-intense part was the brightness and contrast correction of the regional mosaics and the remaining single images by tonematching at the transition zones. The global Ganymede mosaic can be downloaded at https://janus.dlr.de/ and is archived at PSA: DOI 10.5270/esa-mqhvfjf.

\section{Outlook}

The new Ganymede basemap with a global map scale of $359 \mathrm{~m} / \mathrm{pxl}$ including some high-resolution images from Galileo increases the variety of available data products and should help during pre-JUICE arrival investigations of Ganymede and support the planning process.

\section{References}

[1] Grasset et al., 2013, Planetary and Space Science, 78, 121, DOI: 10.1016/S0032063312003777. [2] Zubarev et al., 2016, Solar System Research, 50, 5, 352-360, DOI: 10.1134/S0038094616050087. [3] Kersten et al., 2021, resubmitted to Planetary and Space Science. [4] Zubarev et al., 2015, Planetary and Space Science, 117, 246-249, DOI: $10.1016 /$ S0032063315002007. 